IOS Press

\title{
Advancing new collaborative mechanisms for the profiling of MNEs in national, regional and global group registers
}

\author{
Fred Demollin ${ }^{\mathrm{a}}$ and Hank Hermans $\mathrm{b}^{\mathrm{b}, *}$ \\ a Program Manager Large Enterprises, Statistics Netherlands, The Netherlands \\ ${ }^{\mathrm{b}}$ Deputy Director Economic and Business Statistics and National Accounts, Statistics Netherlands, The Netherlands
}

\begin{abstract}
The importance of Multi National Enterprise Groups (MNEs) on the economy is ever-growing and at the same time, it becomes more complex to capture their activities and structures accurately in official national statistics. The establishment of Large Case Units and the introduction of profiling of MNEs are measures to capture the activities of MNEs correctly so that consistency between statistics can be achieved.

At international level, the same challenges can be found. In Europe the existence of the European Statistical System and accompanying legal frameworks make it possible to organize European collaboration, resulting in a EuroGroups Register and European profiling and the Early Warning System.

The benefit from a Global Group Register (GGR) seems evident: providing unique identification of MNEs and insight in the structure of internationally operating MNEs helps to create valuable information for policymakers on many different economic themes. At a global level, we do not have legal facilities like those in the EU, which makes it important to look for other solutions. An initial GGR has to be built upon publicly available sources and upon sources from commercial data providers. The benefits of establishing a Global Group Register are multiple and work in this area should be encouraged.
\end{abstract}

Keywords: Multi National Enterprise Groups (MNEs), large case units, globalization, statistical business register, global group register, profiling

\section{Introduction}

Statistical Business Registers are the backbone for producing business and economic statistics that meet the increasing demand for better integrated, coherent, and comparable statistics across countries and statistical domains. Statisticians nowadays are facing challenges related to new phenomena like globalization, digitalization, well-being, sustainability and so on. With these new demands, an inclusive and exhaustive Statistical Business Register becomes an increasingly more important element of the statistical infrastructure for maintaining the relevance, responsiveness, and quality of economic statistics in order to measure the structure and dynamics of economic activity. These structures and dynamics are mainly dominated by the occurrence

*Corresponding author: Hank Hermans, P.O. Box 4481, $6401 \mathrm{CZ}$ Heerlen, The Netherlands. E-mail: hjcm.hermans@cbs.nl. and cross border activities of Multi National Enterprise Groups (MNEs). The production of high quality statistics therefore depends in a large amount on the quality of structures of MNEs captioned by an SBR and the capability of measuring and understanding the behavior of those MNEs. This is a challenge on national level, but even more on global level. In this paper we want to touch some elements on national and international level that can be of help in better captioning and understanding MNEs.

\section{The importance of multi national enterprises for statistics}

Multi National Enterprise Groups have gained enormous importance in the world trade and production. The share of MNEs in business statistics represents roughly $40-60$ percent of the added value of the busi- 
ness economy in most countries. Statistics without adequate figures of MNEs are therefore meaningless and unusable.

The awareness within National Statistical Institutes (NSIs) of the importance of correct MNE figures has increased over the past years. A number of NSIs have set up special departments or organizational structures to monitor the information received from large MNEs in a separate (or supplementary) way from the normal data collection and analysis processes.

Special attention is given to the complexity that is introduced by the ongoing globalization within MNEs and the difficulty to administrate the proper figures in the proper economy.

Introduction of the Balance of Payments Manual 6 and European System of Accounts 2010 made it necessary to reconsider ongoing processing and analysis processes. The reconsideration of economic ownership created a new view on the processing of economic statistics.

For more than 15 years the Netherlands are giving special attention to the consistency of MNE information. Around 2000, Dutch NSI got notice that companies with a multitude of contact persons and delivering a big variety of economic statistics show consistency problems in the information they deliver to the NSI. The internal organizational structure of the NSI was stove pipe oriented with a result that consistency problems would become visible at a very late stage in the statistical process namely at the production of the National Accounts. As this moment is roughly one and a half to two years after data collection, all primary statistics (from the stove pipes) are disseminated, and the opportunities to properly consult companies on the consistency of the data are nearly gone.

Various attempts of solving this issue lead eventually to the introduction of a Large Case Unit (LCU) as an individual department within Statistics Netherlands.

\section{Large Case Unit}

Large Case Units are units within a National Statistical institute dedicated to delivering consistent microdata on large and complex enterprise groups (EG) for a number of statistics.

There is a growing awareness that relationships with large enterprise groups need to be maintained very thoughtfully and data, being sent by multiple contacts within these groups, needs accurate checking on consistency.
Ever since the first years of the present century, Statistics Netherlands is experimenting with operations for MNEs only. The start for these activities was the signal from the National Accounts department of Statistics Netherlands, stating that inconsistencies were growing in the data on micro level. National Accounts found out that the problem was the worst with MNE data.

Various attempts were done to set up a sustainable process for the processing and analysis of MNE data. Among them was an attempt to collect data centrally at the level of a head office of an enterprise group. During a pilot 'central data collection' it showed that the availability of data at central level varies per MNE. Some MNEs had fairly comprehensive data available on a central level where other MNE tend to collect only aggregate data on a central level. For this last category of MNEs it was impossible to respond to the regular questionnaires on a central level. The data collection needed to be done on a decentralized level, closer to the operations of the MNE.

As a result of all the test experiences it was decided to keep the data collection unchanged, but to bring all the MNE data as soon as possible together within Statistics Netherlands. The LCU started with a panel of 330 groups and the department was equipped with a software system called 'the consistency system'. This system still forms the heart of the LCU operations. In this system, key variables from all available data collections of individual economic statistics are combined. Next step is that key variables are compared with each other according consistency rules. Whenever the rules detect a difference out of the control limits, an alarm is generated towards the staff, responsible for the concerned MNE within the LCU department. The next step is to analyze the data and to appoint the inconsistencies. All available information will be studied before contact is taken with the MNE itself.

In most cases, the contact is only necessary to verify the proposed actions that were planned according to the desk analysis. In some cases, the contact is necessary to clarify the nature of the inconsistency. Difficult cases sometimes require visits to the involved MNE. During such a visit the inconsistencies can be discussed in depth. The advantage of such visits is that solutions are more sustainable for the future. The MNEs build up their experience and will incorporate these experiences in future data deliveries to the NSI. However, the weak point is that contact persons with MNEs can change; the experience on the side of the NSI learns that whenever a contact person changes, most of the tailor-made solutions with MNEs have to be rebuild. The logging 


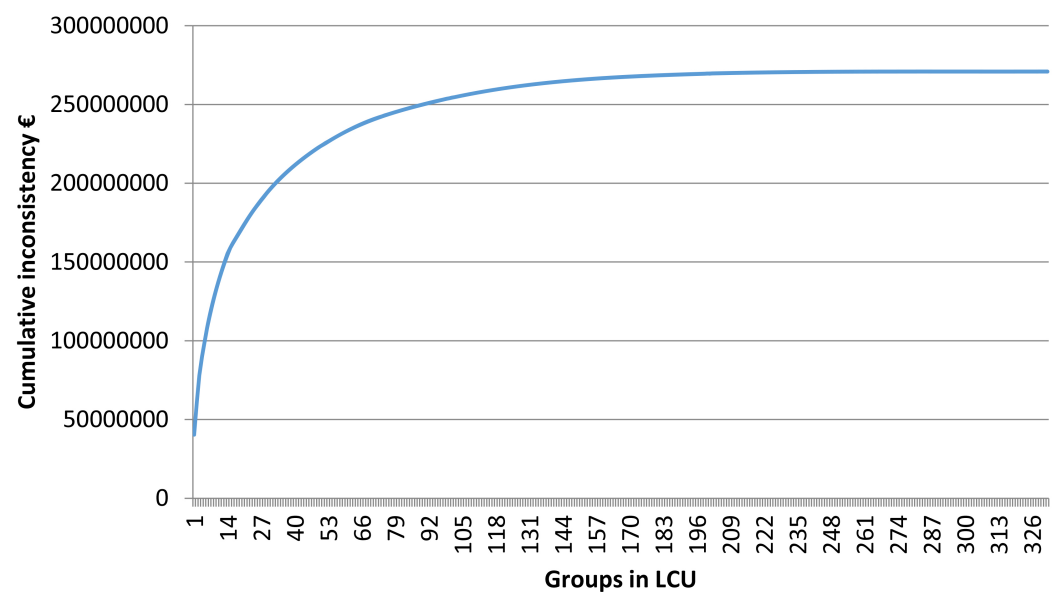

Fig. 1. Cumulative inconsistency in LCU.

and handover of agreements between NSIs and MNEs turn to be very poor. Documentation of special agreements with MNE on the side of the NSI is helpful to re-instruct the new contact person but the instruction of new contacts remains necessary. The changeover of contact persons with the MNEs is therefore an immediate alert towards the NSI to thoroughly check all data from the involved MNE.

The number of Groups in the LCU in the Netherlands was determined on a combination of a selection of largest groups (based on turnover, number of employees and balance sheet total) and a handmade selection of smaller but iconic groups in the Dutch economy. During the establishment of the LCU, the population was regularly monitored in terms of 'most inconsistent' and 'most important for the Dutch economy'. The outcome of this is shown in Fig. 1.

The figure shows two aspects of the LCU. In the first place, there is a good evidence that the most inconsistent Groups are dealt with by the LCU; 80 percent of the inconsistencies were caused by 20 percent of the Groups. In the second place, the figure shows that expanding the size of the LCU has limited additional value for the NSI. The extra efforts for consistency checks on additional Groups will cause little change in the data. Only exceptional cases for that reason are added to the LCU nowadays.

\section{Profiling of MNEs}

\subsection{Knowledge about observation units}

Large MNEs are quite volatile in their organizational structures. Due to their multinational presence, their knowledge about possibilities to exploit local differences in the MNEs interest is enormous. Worldwide competition drives them to take fast decisions in organizational chains, production chains, and taxation schemes. Statisticians need to keep up with the volatility of the MNEs in order to produce correct data and to register and report trends in economies. Profiling of company structures is a very strong tool to support this process.

\subsection{What is profiling?}

According to the European business profiling recommendations manual [1] profiling is a method to analyze the legal, operational and accounting structures of a business, at national and world level, in order to establish the statistical units within that business, their links, and the most efficient structures for the collection of statistical data. To put this in other words; the aim of profiling is to understand the enterprise's business model and to translate this into a useful structure for data collection.

Generally spoken three forms of profiling are being seen, depending on the amount of manual work that is spend on the profiling: fully automated, light, and intensive profiling. On the one hand, there is profiling predominantly carried out on sources and by computed algorithms. This form of profiling can be applied to large amounts of smaller enterprises and will give satisfying results as long as the enterprises are limited in their statistical importance in their industries.

On the other side there is intensive profiling carried out by human profilers and involving a multitude of sources including national available (legal) registers, the internet, statistical data of the last years and personal 
contacts with the MNEs like visits and telephone calls. Intensive profiling is applied for MNEs with substantial importance in statistics.

In the middle there is light profiling that is carried out on a largely automated basis. The results are being monitored and if needed are adjusted. In Statistics Netherlands this way of profiling is being used for large MNEs that are not monitored in the Large Case Unit.

Profiling helps to understand the structure and functioning of an enterprise group, leads to adequate statistical units with the group, and finally leads to data collection where enterprises correctly can respond to.

Especially, with large MNEs this last aspect is of great importance; whenever a MNE does not recognize itself in the NSI created collection units, it will be forced to make decisions on what elements of their activities reporting will take place. There is no doubt that these choices will lead to inconsistent data collection and finally to errors in the statistical domain.

Profiling has to verify the ability of enterprise groups to report in the desired way with an acceptable response burden.

\subsection{MNEs benefit from profiling}

As previously explained, profiling can lead to important advantages for NSIs. The reporting units on their turn might also experience advantages from adequate profiling. In the profiling phase a profiler can discuss proposals for an optimal construction of statistical units with the reporting enterprise group. The agreements with MNE are made during conversation with the MNE staff and are based on common sense; no legislation is applied or necessary for this. Special attention should be given to the construction of data collection units that are appropriate for the statistics they are intended to deliver information to. When data collection units are very similar to the units they have in their administrative systems, the effort to respond to the NSI data collection is minimal with a maximum guarantee of delivery of correct and required information. When the structure and the underlying activities and flows within groups are clear to the NSI, even automation of data delivery is possible. The data is delivered according to well designed and tested algorithms. A prerequisite for this is, of course, a correct and up to data structure of the underlying enterprise (group) leading to a correct and complete data collection.

In the near future enterprises might deliver data automatically to the NSI: At present Statistics Netherlands is doing tests with automatic withdrawal of data directly from business administrative systems. By the creation of appropriate statistical units with close relation to the group structure, the information in the administration can be consolidated and reported through computing. In this way the data received by the NSI is not only available earlier, the quality is far better as the potential problems have been detected and eliminated in the test phase. After acceptance of the set up's of the software by the Enterprise group and the NSI. The time saving might add up to 4 months for yearly statistical results.

The computed results replace an amount of manual work that equals some 80 working days for a large MNE. The importance for reporters to statistical questionnaires to cooperate actively to profiling consequently can be considerable. An additional advantage of cooperation in profiling for enterprise groups is reduction in additional burden caused by inquiries of a NSI tracing back inconsistent information.

As NSI profilers and group contacts strive for failurefree reporting through profiling, the necessity to verify reported information after adequate profiling is considerably reduced. Failure free reporting saves considerable amounts of investigation time within the reporting unit and within the NSI. Consistent data will also reduce time needed for repeated reporting to the NSI.

The investment in profiling is not only favorable in time during data collection, data analysis and verification; it increases the image of NSI's in terms of professionalism and accuracy drastically.

The experience within Statistics Netherlands is that especially MNEs are generally very willing to participate in activities to improve quality of delivered data and reduce discussion about consistency in the data. Sensitive subjects as tax regulations are more delicate to discuss but when a trusted relation is build up in time, MNEs proved to be willing to reveal a lot of details. Profiling is an indispensable activity to achieve the prerequisites for proper data collection.

\subsection{Nationality of an MNE}

Nationality of a group can be defined in several ways. The ultimate legal unit in the worldwide legal structure of a group is referred to as the Global Group Head. This legal unit is indeed the ultimate unit in the tree structure of the group but not necessarily employs the influential decision making directors. Tax avoidance of groups leads to worldwide flows between legal units in a global group structure. For this reason, special legal units are created with the sole purpose to maximize profits. These legal units most commonly employ zero employees and 
are referred to as 'empty shells' or 'mailbox companies'. Real decision making is lacking within these involved legal units; the nationality of these legal units is a result of worldwide tax legislation. As the association with tax avoidance is growing unpopular under large groups, groups tend to be careful in elaborating on taxrelated structures. Good relations with group contacts and confidence in the confidentiality of a NSI is crucial in exploring tax-related constructions. A profiler can play a vital role in this process.

The group's legal unit where the main strategic decisions are taken is referred to as the Ultimate Controlling Institutional Unit (UCI). The nationality of the UCI is used to determine, among others, the reporting nationality in Foreign Affiliates Statistics. The decision whether or not to call a group domestic or foreign is based on some specific characteristics.

The location of the group's top management is a first indicator and generally referred to as the Global Decision Centre (GDC). The nationality of the directors is an indication for the nationality of the group as well, however in a further globalizing environment this is just a weak indicator. The location where the general shareholders meeting is held and the currency used in the annual reports give another indication about the nationality of a group.

Generally, the different indicators end up in the same country and the nationality of the group is clear. The most difficult cases are those where all indicators for the nationality of the UCI end up in different countries. In ambiguous cases, consultation with involved NSIs is necessary to reach an agreed final position between the different countries. Unaligned UCI populations lead to double counting or missing elements in statistical reporting.

The top of the group determines for a large amount the contribution to the national GDP and GNI a group has. Furthermore, for EU countries, the initiative to (internationally) profile a group lies in the GDC country. Correct reporting in various statistics is also depending on correct nationality of the involved groups. The importance of good quality of group nationality is evident.

\subsection{Cross border activities of MNEs}

Globalization has seriously influenced the way enterprises organize their activities. National borders are no constraints anymore in the organization of production processes. Groups tend to have different challenges in their production flows like market access, availability and accessibility of raw materials and $R \& D$, avail- ability and cost of personal, tax regulations and legal and environmental circumstances applicable to certain processes. Altogether a lot of choices to be made for MNEs with a considerable effect on national statistics. NSIs have great difficulties in discovering and understanding process flows of MNEs, not to speak about costs and added value involved in the process. Since the introduction of the Balance of Payments Manual 6 and the ESA 2010, economic ownership is determinative in allocation of the added value to national economies. Especially for border crossing internal transfers within MNEs a variety of scenarios has been developed. Ownership can remain unchanged when goods are exported for processing, raw materials can be delivered to subcontractors in foreign countries, transfer prices can be calculated when goods move within a MNE, royalties can be due to other group entities for the use of special processing methods or technical support. All these actions have a specific effect on GDP calculation of a national economy. To produce a high quality GDP, it is necessary to understand border crossing operations of MNEs and to incorporate the correct proportions of the activities in national statistics and consequently in national accounts.

Understanding material and financial flows in MNEs starts with a comprehensive profile of the involved MNE. Profilers, as being generally the first contacts to large groups, have an important function in discovering the ins and outs of the MNEs production processes and the financial flows concerned with these processes. Knowledge about the operations of MNEs can be used by profilers to delineate correct statistical units in a MNE structure. In depth knowledge about production processes helps in the determination of observation units fit for collection of the appropriate data from MNEs and finally to correct statistical information on national level.

\subsection{Need for profiling in business statistics}

Traditionally, NSIs used to organize their production processes in stove pipes: separate statistical production processes organizing all activities from the determination of the involved population through the development of relevant questionnaires to the publication of the final data.

Nowadays, most NSIs use exhaustive business registers as a common backbone for a multitude of statistics. A business register is a prerequisite for adequate coordination between statistics and integration possibilities of statistical results. 
With the consequent use of business registers also the provision of information within the statistical production processes has achieved a next level. Information from the beginning of the chain can easily be passed through to other parts of the chain. Statisticians usually send out their questionnaires with some delay after the involved statistical period has been closed. Whenever they detect abnormalities in the data they collect, it is a challenge to get in contact with MNE representatives that can (re)produce the proper details on the underlying statistical period. In these situations, thorough fully administered profiles help in understanding the observed data.

As profilers operate far beyond the rest of the NSI, their reports about important moves in structures of MNEs and their knowledge about production processes and financial flows create fundamental information in the analysis processes. The challenge is to administer the profile information in a way that is findable, accessible and understandable for the rest of the NSI. The profile information is very often more than one year old before others within the NSI tend to make use of it. Administration on correct reference numbers of MNEs and in easy to use database structures is a necessity for meaningful use of the information. Feedback possibilities on the supplied information are inevitable to secure continuous improvement in the work of the profilers and to guarantee the fact that the proper details are being asked to MNE contacts during visits and telephone calls.

\section{Collaboration at national level}

In previous chapters we have mainly focused on the activities within an NSI concerning MNEs. We have discussed the importance of MNEs for national statistics and the need for an NSI to understand what is happening within an MNE. The establishment of an LCU and the focus on consistent data of an MNE across statistics leads to a set of consistent data within the NSI, reflected in official statistics. But often an NSI is not the only producer of statistical information at national level, other national institutes may also produce statistical information that may differ from published figures from an NSI, leaving the reader with questions behind. So reaching out for higher quality of statistics at national level requires also consistency at national level and hence, collaboration between producers of statistical information should be stimulated.

In the Netherlands for example, the consistency between the Balance of Payments/International In- vestment Position (BOP/IIP) and the rest-of-the-world (ROW) account as part of the national accounts has become an important area of concern [2]. Differences between these areas were often caused by differences in the sources used despite the fact that these sources may origin from exactly the same MNEs. To reduce or even eliminate the differences between BOP/IIP and ROW account, Statistics Netherlands (responsible for the compilation of the Dutch national accounts including the financial accounts) and the Dutch Central Bank (responsible for compilation of BOP/IIP) have intensified their cooperation. Previously the data received from MNEs by both institutions were compared and analyzed so that differences could be explained and solved. Now, the populations of non-financial enterprises are fully aligned and a joint survey for finance of enterprises and balance of payments has been developed. This survey is sent to the 350 largest enterprises in The Netherlands and since 2019 these enterprises now only report to Statistics Netherlands. The microdata received is analyzed and edited by Statistics Netherlands and then shared with the Dutch Central Bank. The result is that figures of BOP/IIP and ROW account now are fully aligned.

Next step in this cooperation that is planned for 2020 is the transition to one single Statistical Business Register (SBR) serving both institutions. In concrete terms this means that the current SBR of Statistics Netherlands will be perfected in the field of information about financial institutions. This also implies that profiling of financial institutions will be a joint responsibility. Profiling of non-financial enterprises will remain the responsibility of Statistics Netherlands, but the exchange of information about those enterprises in order to build up an overall accurate picture of MNEs will be a joint responsibility. One single SBR with the enterprise group as key statistical unit will be functioning as backbone for all official business statistics.

\section{International collaboration}

\subsection{European profiling}

The increasingly global activities and structures of enterprises challenge the integration, coherence, consistency and comparison of business- and macroeconomic statistics across countries and across statistical domains. Having all measures in place for achieving consistency at national level, like an LCU, profiling and even collaboration with other national authorities, 
new challenges arise when looking at the international picture. Take for example the European territory: when adding up national figures to European totals we want to be sure that there is no chance at double counting or missing figures due to the possibility that MNEs are not completely covered or due to cross border events that are not recorded at the same time at the same way in countries concerned. These challenges cannot be tackled easily and ask for measures at different levels.

To get a clear picture how enterprise groups organize their production chains across national boundaries the profiling of large and complex enterprises that are part of multinational enterprise groups benefits by cooperation between countries. Different countries may well have a common interest in gaining a better understanding of the global structure of a multinational group with significant economic activity in their country because the relocation of activities and assets from one country to another can have an impact on the correctness of the statistics of all countries involved. In such cases, the relevant information may be not available at national level or it may be extremely difficult to validate. As a result, no country alone can make adequate progress and ensure high data quality. With collaborative international profiling, the country in which the global decision center of a multinational enterprise group is located could initiate the profiling process and coordinate with the countries where the group has affiliated enterprises [3]. In an ideal situation, countries would share their information with each other so that all pieces together help to get a clear and complete picture of an MNE. In practice, however, confidentiality and national legislation restrictions limit the possibilities for sharing data leading to an extra challenge in creating the complete picture.

\subsection{Euro groups register}

At national level, the results of profiling of MNEs are stored in the national Statistical Business Register, providing the backbone for economic and business statistics. In the international context it is also desirable to have a structured solution where cross border relationships and activities of the most important multinational enterprise groups (MNE) can be stored, maintained and made available for compiling statistics on cross border phenomena.

In the European context, the Euro Groups Register (EGR) is the joint tool in the ESS for the coordination of basic information about MNEs from the EU Member States' Statistical Business Registers (SBR). Based on commercial sources and input from the Member States the EGR links and processes data creating the global structures of MNEs resident in the EU and their constituent legal units. This process leads to continuously improved information in the EGR.

The implementation of the EGR is made possible by a legal framework on data sharing within the European Statistical System. The EGR assures unique identification of legal units, global groups and global enterprises. Together with the relations between these units, the EGR supplies a backbone for statistics on cross border effects. Next to that, NSI can check and compare characteristics in their national SBR and in that way achieve completeness and accuracy in their national frames.

\subsection{Early warning system}

Within Europe it became clear that the effects of changes in MNE structures most commonly are not restricted to one sole country. The examples where restructuring in one country, leads to effects in other countries are numerous. As the ESS aims to be a coordinated system for the whole European Union it is necessary to discuss large restructuring across borders. This lead to the establishment of the Early Warning System (EWS); a fully secured system where countries share knowledge between involved countries in restructuring events [4]. The country that receives the first information on events can open up a case among the involved countries. Sharing all available knowledge between the NSI leads to a coordinated processing of the event in the involved countries. The EWS leads to an exchange of early profiling information as well as foreseen effects on the data. Surprises for statisticians are tackled before they could pop up.

\subsection{European GNI MNE pilot}

The benefits of having an EGR and the use of profiling became clear during a pilot on multinational enterprises that was carried out by the Member States of the European Union in 2018 and 2019. The goal of this pilot was to understand the reliability of the recording of globalization in Gross National Income (GNI) data. The pilot concerned $25 \mathrm{MNE}$ cases selected on the basis of a materiality threshold and the wish to involve all EU Member States. The NSIs and NCBs were participating and cooperating on the most prominent issues such as: checking the complete structure of the MNE group in the EU in terms of legal units (missing or double counted) and their impact on value added; economic 
versus legal ownership of products and assets and treatment of intangible property products and research and development.

All participants accepted to voluntarily share confidential data on an unprecedented scale on a need to know basis and according to the "Code of Conduct on Micro-Data Sharing", prepared in the context of the GNI MNE pilot. Confidential data were stored in a secure environment.

The GNI MNE pilot exercise showed clearly the benefit for increased cross-domain, cross-country cooperation and information sharing on resolving and analyzing statistical data on MNEs. Understanding the group structure was much easier for those MNEs that were recently profiled. The pilot also showed that MNE with important links to or GDC situated in an Extra-EU country calls for information on cross border relations, MNE activities and structures at global level [5]. We therefore want to elaborate on possible steps in a global perspective.

\subsection{Global group register}

Activities of MNEs are not bound to the European borders and in the ideal situation a Global Group Register (GGR) that at least could serve statistical purposes would be very welcome. A future GGR should focus on its comparative advantages to the national SBRs, providing unique identification and insight in the structure of internationally operating MNEs that cannot - or only with huge efforts - be achieved solely at national level. Its role to fulfil is connecting national master frames in SBRs of countries worldwide by allowing linking information from all the national SBRs. With a GGR recorded structures and links among enterprises in different countries show how control is exercised [6]. The United Nations Statistical Commission, at its $46^{\text {th }}$ session in 2015, mandated the development of a GGR [7]. UNSD is actively working toward the development of the GGR in collaboration with the Task team on Exhaustive Business registers of the Committee of Experts on Business and Trade Statistics and building on existing initiatives such as the EGR of Eurostat and ADIMA of OECD.

Ideally such a register is built in accordance to the spine-model [8] that is being used in Australia and using a unique identifier for MNEs so that information on national level can be linked to the register on global level, lets new opportunities arise. We will be able to combine data from many different sources turning it into valuable information for policy makers on many different economic themes [9].
A first hurdle to take will be the sharing of microdata. Sharing confidential data on such a wide scale is not likely to happen, knowing that a legal framework such as the one in the European Union will not be possible. This implies that information in the GGR initially has to be built upon publicly available information and information from commercial sources. A starting point could be to make a list of available data providers in the public and commercial domain and select one to serve as primary base. The big challenge after that will be to motivate the NSIs to validate and supplement the contents of the GGR so that its quality will increase in time. Once again, initiatives like the EGR and ADIMA could be great starting points and an initial goal could be to cover the largest MNEs worldwide. Once it has proven its usefulness the coverage of the GGR could stepwise be enlarged.

\section{Closing remarks}

Paths arise by walking them so they say. In this paper we discussed the different measures on national level to obtain consistent data sets from MNE leading to higher quality of official statistics. At European level collaboration by NSIs within the European Statistical System is facilitated by a sound legal framework leading to a EuroGroups Register maintained by European profiling carried out by the NSIs. As the activities of MNEs are not bound to any border whatsoever and the need for an overall picture of MNEs and their cross border relationships and activities is growing by time, the need for a global group register seems evident. It is clear that the circumstances differ very much from the European situation, and we are certainly able to come up with innumerable hurdles to tackle, but sometimes it is better just to start doing. The benefits of establishing a Global Group Register are multiple and work in this area should be encouraged.

\section{References}

[1] European business profiling, recommendations manual, 2020 edition, Eurostat, February 2020 (https://ec.europa.eu/eurostat/ en/web/products-manuals-and-guidelines/-/KS-GQ-20-002? inheritRedirect $=$ true $\&$ redirect $=\% 2$ Feurostat $\% 2 \mathrm{Fen} \% 2 \mathrm{Fpublica}$ tions\%2Fmanuals-and-guidelines).

[2] Developing a new approach to compiling the balance of payments in the Netherlands, Erik Bieleveldt and Pim Claassen, Dutch Central Bank, October 2014.

[3] The European profiling of multinational enterprise groups: a crucial tool in the current European developments on statistical units to improve national and European business statistics, Dominique Francoz. 
[4] BSDG 2019063.4 - Systematic and coordinated approach to MNEs, Eurostat, Business Statistics Directors Group - Strategic Development Group (June 2019).

[5] Final report on globalisation $-2^{\text {nd }}$ Meeting of the GNI expert group, Eurostat, November 2019.

[6] Developing a Global Statistical Register for Multi-National Enterprise Groups (25th Meeting of the Wiesbaden Group on Business Registers), Amerigo Liotti and Ronald Jansen, November 2016.
[7] Report on the forty-sixth session (3-6 March 2015) page 21, Statistical Commission, United Nations, New York, 2015 (https://unstats.un.org/unsd/statcom/46th-session/ documents/statcom-2015-46th-report-E.pdf).

[8] Towards a more flexible ABS Business Register, presentation at UNCEBTS June 2019 meeting in New York - session 5, ABS Luisa Ryan (June 2019).

[9] Discussion paper: a dot on the horizon and a possible way forward (Task Team on "Exhaustive Business Registers" - UN Committee of Experts on Business and Trade Statistics), Hank Hermans, September 2019. 